\title{
Light-addressable lab-on-a-chip based analysis platform
}

\author{
R. Welden ${ }^{1}$, L. Breuer ${ }^{1}$, B. Schneider ${ }^{1}$, M.J. Schöning ${ }^{1,2}$, T. Wagner 1,2 \\ 1 Institute of Nano- and Biotechnologies, FH Aachen, Campus Jülich, Heinrich-Mußmann-Str.1, 52428 \\ Jülich, Germany \\ 2 Institute of Complex Systems (ICS-8), Forschungszentrum Jülich GmbH, Wilhelm-Johnen-Str., \\ 52428 Jülich, Germany \\ torsten.wagner@fh-aachen.de
}

\begin{abstract}
During the last decades, research on lab-on-a-chip systems has continuously increased. Today's systems have multiple functionalities on a single chip, like flow control, triggering and detection of analyte reactions or stimulation of cells. To enhance flexibility and perform different tasks in a single system, light-addressable technologies can be used. E.g., it is possible to use light-addressable hydrogels for flow control, light-addressable potentiometric sensors for sensing and light-addressable electrodes for stimulation inside measurement chambers. These technologies have to be adapted and combined for the integration in a common set-up. In this work, the single technologies will be introduced and the actual progress of combining these technologies into a common analysis platform will be presented.
\end{abstract}

Key words: lab-on-a-chip, microfluidics, light-addressable hydrogels, light-addressable potentiometric sensors, light-addressable electrodes

\section{Introduction}

In today's medicine, easy and quick diagnostic tools play an important role. Lab-on-a-chip systems with integrated microfluidics can have the functionality of a miniaturized analysis system combining several functionalities on a single chip which are normally performed at laboratory scale. It is possible to control the flow, measure specific analyte concentrations and to manipulate the sample (e.g., perform electrolysis). However, these platforms are often complex, due to the highly sophisticated structures of integrated sensors and actuators. To decrease the complexity, the integration of sensors and actuators, which can be stimulated and triggered by light, can be one possible approach. Therefore, light-addressable hydrogels [1], light-addressable potentiometric sensors [2] and light-addressable electrodes [3] have been integrated in one common analysis platform (Fig. 1)

\section{Light-addressable hydrogels}

For active flow control, e.g., to address individual measurement chambers, lightsensitive hydrogels can be integrated as valves within the microfluidic system. One possible approach is the use of temperature-responsive

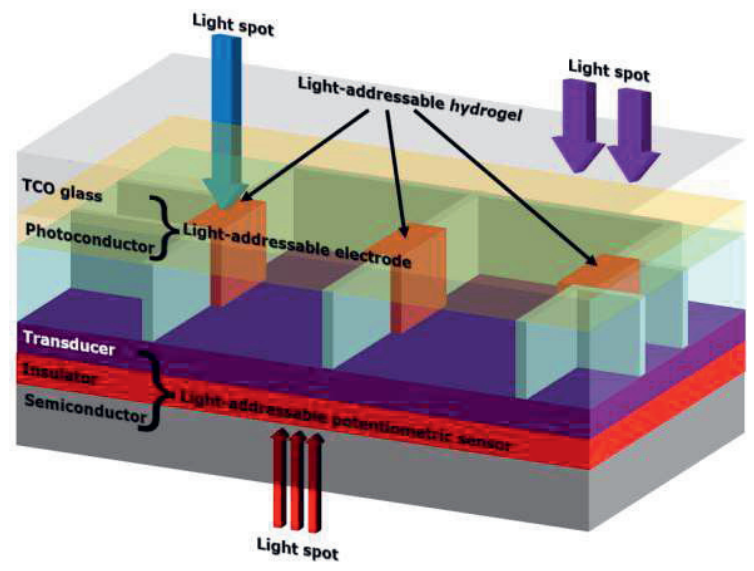

Fig. 1 Schematic illustration of a microfluidic structure with embedded light-addressable hydrogels for flow control, a light-addressable potentiometric sensor for sensing (e.g., cell metabolism) and a lightaddressable electrode for manipulation (e.g., electrolysis) inside micro-chambers.

Poly-N-isopropylacrylamide hydrogels with integrated graphene oxide (GO) nanoparticles. Temperatures lower than the lower critical solution temperature will lead to a swelling of the gels in water. Though, due to the increased light-absorption by the GO nanoparticles, the gels can be heated and stimulated optothermically, which will lead to a contraction. 
Therefore, swollen hydrogels are able to block microfluidic channels. Upon illumination, they will undergo a volume change (water will be released) and can open the channel.

\section{Light-addressable potentiometic sensors}

Light-addressable potentiometric sensors consist of a semiconductor, insulator, transducer sandwich structure. Applying a d.c. bias voltage, a space-charge region in the semiconductor will be generated. Furthermore, illumination of the rear-side with a modulated light source will generate a photocurrent. Due to local variations of the analyte concentration, the Nernstian potential at the sensor surface will change locally. This will influence the width of the space-charge region and therewith the photocurrent. Moving the light source in a raster-like manner underneath the sensor surface, it is possible to record spatially resolved the resulting photocurrent and hence the variations of the analyte concentration. A chemical image e.g., $\mathrm{pH}$ changes within a microfluidic cell, can be displayed by matching all measurements into a single image.

\section{Light-addressable electrodes}

For the manipulation inside microfluidic channels, e.g., to adjust the $\mathrm{pH}$ value or perform cell stimulations, light-addressable electrodes can be used. Those electrodes are based on semiconducting materials. For rearside illumination, the semiconductor is deposited on a transparent, conductive glass. The semiconductor is in direct contact with the analyte. Illumination of the electrode with a suitable light source will lead to the generation of charge carriers. Due to the photoelectric effect, the illumination will result in an area with high conductance. A localized photocurrent will occur while the remaining electrode surface is still insulating. Thus, it is possible to adjust the geometry and the position of the electrode area in-situ.

Integrating all technologies in a common platform requires a careful adjustment of the individual parameters, so that they will not influence each other. Thus, a programmable light source can be used to determine which function at which position within the lab-on-achip system should be executed. This novel platform forms a modular system which should be available for research and industry and enables high flexibility and low production cost.

\section{Acknowledgements}

This work was supported by the German Federal Ministry of Education and Research (BMBF) within "NanoMatFutur" (13N12585)

\section{References}

[1] L. Breuer, M. Raue, M. Strobel, T. Mang, M.J. Schöning, R. Thoelen, T. Wagner, Hydrogels with incorporated graphene oxide as light-addressable actuator materials for cell culture environments in lab-on-chip systems, physica status solidi (a) 213(6), 1520-1525 (2016); doi: 10.1002/pssa.201533056

[2] T. Wagner, C.F. Werner, K. Miyamoto, M.J. Schöning, T. Yoshinobu, Development and characterization of a compact light-addressable potentiometric sensor (LAPS) based on the digital light processing (DLP) technology for flexible chemical imaging, Sensors and Actuators B: Chemical 170, 34-39 (2012); doi:

10.1016/j.snb.2010.12.003

[3] J. Suzurikawa, M. Nakao, R. Kanzaki, H. Takahashi, Microscale $\mathrm{pH}$ gradient generation by electrolysis on a light-addressable planar electrode, Sensors and Actuators B: Chemical 149, 205-211 (2010); doi: 10.1016/j.snb.2010.05.058 